\title{
Ultra-fast, high-density 3D mapping system for catheter ablation of infrequent, "unmappable" idiopathic ventricular arrhythmia - a case report.
}

\author{
Radosław Lenarczyk ${ }^{1, A-F}$, Oskar Kowalski, A-C, E-F, Michał Mazurek ${ }^{1, \text { D-E }}$, Zbigniew Kalarus ${ }^{1, A, C, E-F}$ \\ A - Research concept and design, B - Collection and/or assembly of data, C - Data analysis and interpretation, \\ D - Writing the article, E - Critical revision of the article, F - Final approval of article \\ 1. Dpt of Cardiology, Congenital Heart Disease and Electrotherapy, Silesian Center for Heart Disease, ul. Curie-Sklodowdskiej 9 \\ Zabrze, Poland
}

Address for correspondence:

Radosław Lenarczyk, Associate Professor, Dpt of Cardiology, Congenital Heart Disease and Electrotherapy, Silesian Center for Heart Disease ul. Curie-Sklodowdskiej 9 Zabrze, Poland, email: radle@poczta.onet.pl

Oskar Kowalski, Associate Professor, Dpt of Cardiology, Congenital Heart Disease and Electrotherapy, Silesian Center for Heart Disease ul. Curie-Sklodowdskiej 9 Zabrze, Poland, email: oskowa@vp.pl

Michał Mazurek M.D., Dpt of Cardiology, Congenital Heart Disease and Electrotherapy, Silesian Center for Heart Disease ul. Curie-Sklodowdskiej 9 Zabrze, Poland, email: m.i.c.h.a.l@wp.pl

Zbigniew Kalarus, Professor, Dpt of Cardiology, Congenital Heart Disease and Electrotherapy, Silesian Center for Heart Disease ul. Curie-Sklodowdskiej 9 Zabrze, Poland, email: zbigniewkalarus@kalmet.com.pl

\section{Abstract}

A 39-year-old woman with symptomatic, idiopathic left ventricular extra beats was scheduled for a redo ablation. During the procedure the arrhythmia appeared to be very infrequent (1 extra beat/min), making it practically "unmappable" for the conventional mapping systems. An ultra-fast, high-density 3D mapping system (Rhythmia, Boston Scientific, Cambridge, MA, USA) with a 64-electrode basket mapping catheter (Intelamap Orion) was used to successfully tackle the arrhythmia.

\section{Key words:}

catheter ablation, idiopathic ventricular arrhythmia, high-density mapping, basket mapping catheter

\section{Background}

Non-sustained or hemodynamically unstable arrhythmias are usually extremely difficult to map or may even be "unmappable", thus making them virtually impossible to tackle with catheter ablation. The Rhythmia navigation system (Rhythmia, Boston Scientific, Cambridge, MA, USA) is a novel system that allows for ultra-fast, high-density 3D mapping of various cardiac structures. Preliminary reports suggest the feasibility and efficiency of this method, although data on its application in various clinical scenarios are still scarce (1-2). Here we report a patient scheduled for ablation of frequent, symptomatic and idiopathic ventricular extra beats along with non-sustained ventricular tachycardia, in whom arrhythmia virtually disappeared after sedation and insertion of electrodes, thereby making it practically unmappable. Use of the Rhythmia mapping system was found to be very helpful and efficient in this scenario.

\section{Case report}

A 39-year-old woman with recurrent, symptomatic and idiopathic, non-sustained left ventricular tachycardia (nsVT) and ventricular extra beats (VEB) was admitted for a redo ablation. The first procedure was performed in 2012 due to a frequent (24 000 extra beats and numerous nsVTs/24 h) and very symptomatic arrhythmia, originating from the left ventricle. A successful procedure was performed with a 3D mapping system, namely CARTO, resulting in the patient being asymptomatic until very recently. Half a year ago palpitations reoccurred and 24-h Holter ECG monitoring captured over 14000 monomorphic ventricular extra beats. In addition, 12-lead ECG showed extra beats with right bundle branch block-like morphology, with positive ventricular complexes in leads I, II and aVL, and negative in lead III, along with a transitional zone in lead V5 (Figure 1). Echocardiography showed normal size and function of the left and right heart, with no significant valvular lesions. Consequently, the patient was scheduled for a redo procedure.

The procedure was performed under sedation (opioid and benzodiazepine) and local anesthesia. Mapping of the left ventricle and subsequent ablation were attempted based on VEB morphology in both the underlying ECG and arrhythmia morphology captured during the previous ablation. Once the vascular access sites were ready (left femoral vein and right femoral artery) and the intra-cardiac electrode had been placed 


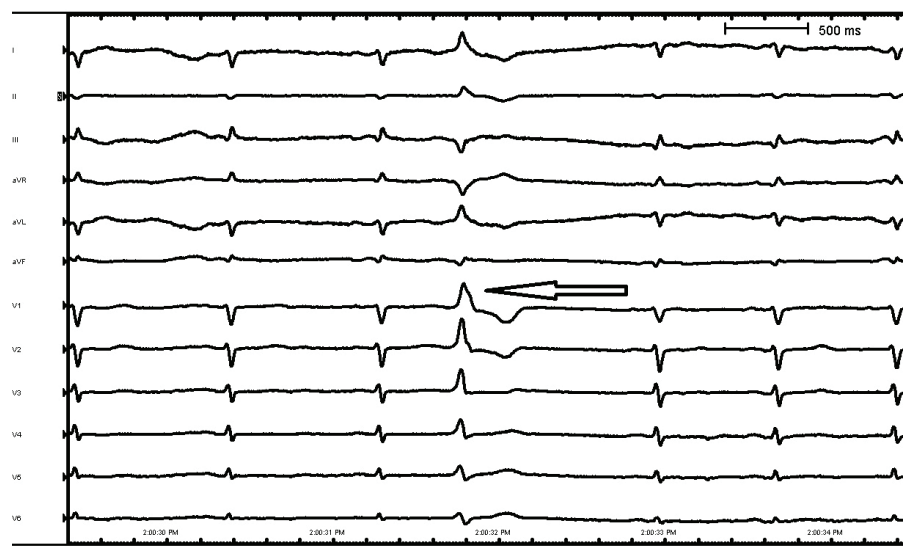

Figure 1. Standard 12-lead ECG, normal sinus rhythm with target extra beat (marked with arrow)

in the right ventricle, the arrhythmia became very infrequent (only approximately 1 extra beat per min). Right ventricle pacing prior to and after isoproterenol infusion also failed to significantly increase arrhythmia frequency. A decision was made to use an ultra-fast, high-density 3D mapping system (Rhythmia). A nine-F vascular sheath for the right femoral artery was used to introduce an $8.5 \mathrm{~F}$ basket mapping catheter (Intelamap Orion, Boston Scientific) into the left ventricle (transaortic route). The Orion mapping catheter is a deployable basket electrode (deployment range 3-22 $\mathrm{mm}$ ) equipped with 64 electrodes (Figure 2), and thus capable of acquiring signals

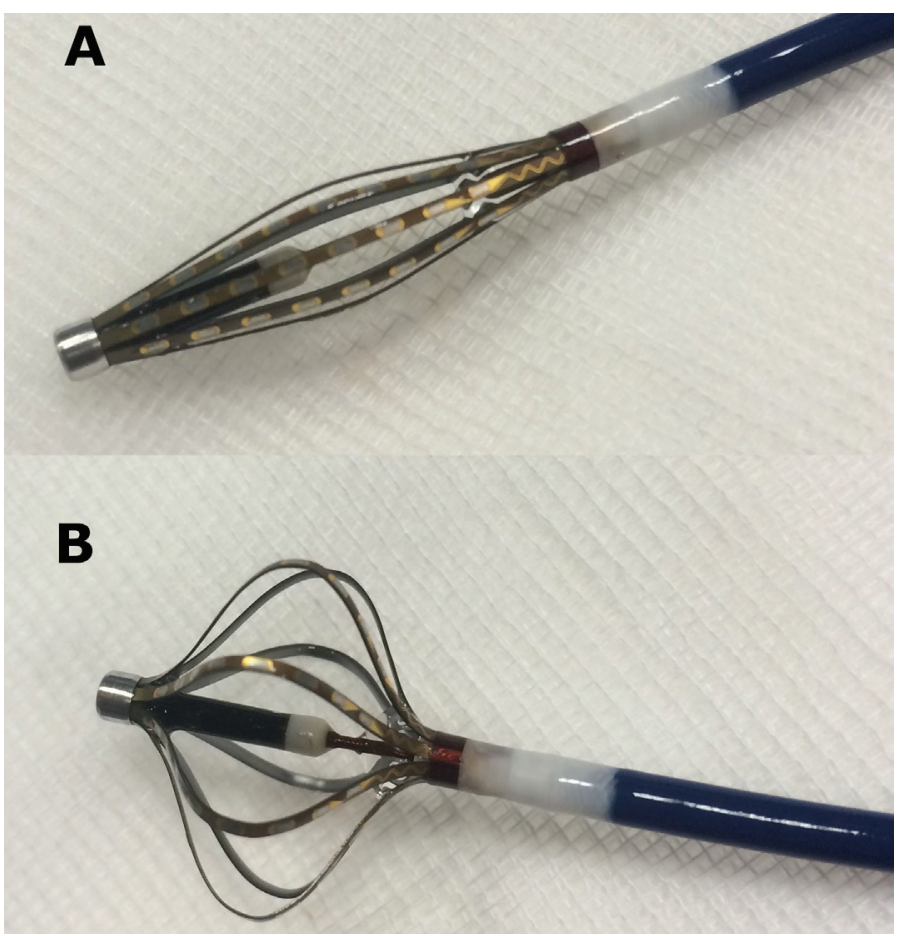

Figure 2. Multielectrode basket Orion mapping catheter. Panel A - basket closed, panel B - basket fully deployed (diameter $22 \mathrm{~mm}$ )

simultaneously from 64 different points of the cardiac cavity at one heartbeat. Although during a 65-minute left ventricular mapping only 75 extra beats were observed, this was sufficient for the Rhythmia system to collect 624 intra-cardiac electrograms and to draw an activation map. This comprised sites of the earliest activation (area of interest) - figure 3. Pacemapping confirmed the location of arrhythmia origin. Using an irrigated tip ablation catheter, eight RF applications were delivered at

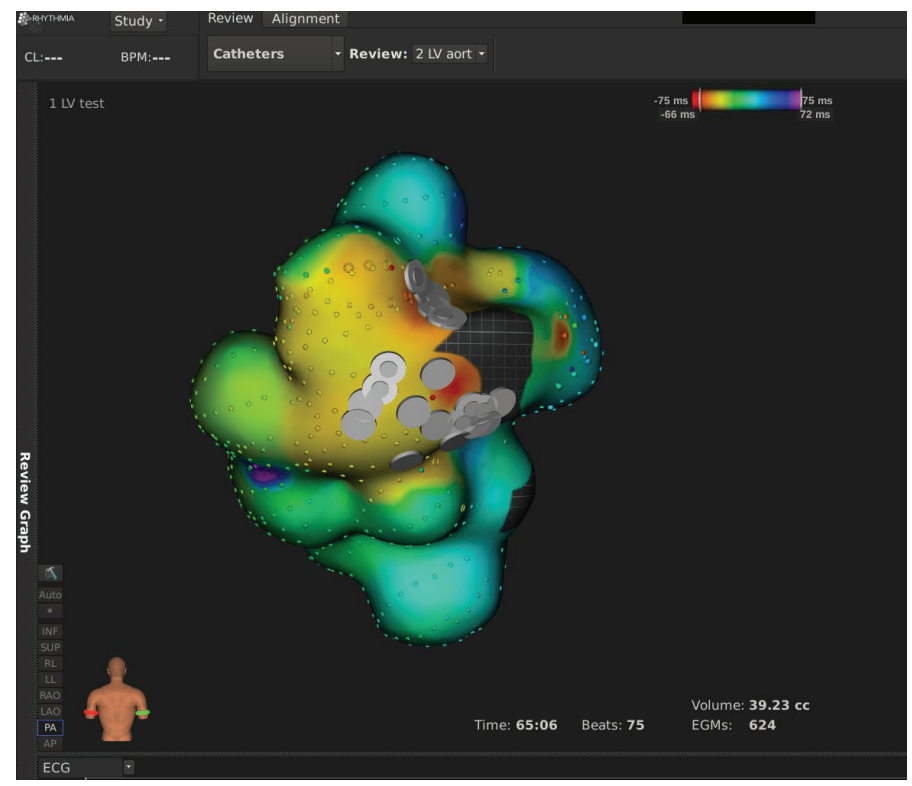

Figure 3. Posterior-anterior (PA) view on a 3D high-density activation map of the left ventricle. Red color denotes the earliest activation, violet denotes the latest one. NB: the map represents only a small part of the ventricle (with volume of only $38 \mathrm{ml}$ ). Application points are marked as grey dots.

sites of the earliest activation (located relatively close to the posterior papillary muscle), resulting in complete remission of arrhythmia. Right ventricular pacing both in the drug-free state and during continuous infusion of a beta-mimetic (isoproterenol) failed to evoke extra beats. Procedure duration (skin-to-skin) was $1 \mathrm{~h}$ and $38 \mathrm{~min}$. Fluoroscopy duration and exposure were $16 \mathrm{~min}$ and $104 \mathrm{mGy}$, respectively. The procedure and post-procedural in-hospital period were uneventful, and the patient was discharged home on the second day. After one month of observation, the patient remains asymptomatic and free of arrhythmia on ambulatory Holter ECG.

\section{Discussion}

Rhythmia with the 64-electrode Intelamap Orion mapping catheter is a newly developed mapping system for rapid, automatic generation of high-density anatomical, voltage and activation maps of the heart chambers. Its utility has been recently demonstrated, initially on animal models, and subsequently in a wide variety of arrhythmias in humans. These included atrial tachycardia, atrial fibrillation, atrioventricular nodal reentry tachycardia, and ventricular tachycardia in structural heart disease (1-8). Our case shows that the system may be very helpful by generating a detailed activation map despite infrequent arrhythmia at the time of the procedure. By contrast, collecting electrograms by the "point-by-point technique" (e.g. using the CARTO system) would last $>10$ hours if one were to collect over 600 points. However, using the Rhythmia system, which automatically collects over 8 electrograms per heartbeat, this process can be shortened to one hour only. Although our map was confined to selected parts of the ventricle, it was precise enough to allow for successful 
RF ablation. This ultra-fast, high-density mapping may be even more important in patients with severely compromised left ventricle function and electrical storm, in whom arrhythmia is usually hemodynamically unstable, and/or very difficult to induce. In such cases, the ability to build a high-quality activation map quickly may be of particular importance.

Our data are based on a single case observation of limited duration (1 month). Thus, our findings, though appealing, are to be considered as hypothesis-generating only, and further studies are warranted to establish the position of Rhythmia and other new mapping systems in a "real life" clinical setting.

\section{REFERENCES}

1. Anter E, Tschabrunn CM, Contreras-Valdes FM, Li J, Josephson ME. Pulmonary vein isolation using the Rhythmia mapping system: Verification of intracardiac signals using the Orion mini-basket catheter. Heart Rhythm. 2015; 12: 1927-34.

2. Sohns C, Saguner AM, Lemes C, Santoro F, Mathew S, Heeger C, Reißmann B, Maurer T, Riedl J, Fink T, Hayashi K, Ouyang F, Kuck KH, Metzner A. First clinical experience using a novel high-resolution electroanatomical mapping system for left atrial ablation procedures. Clin Res Cardiol. 2016 Jun 11. [Epub ahead of print]

3. Tanaka Y, Genet M, Lee LC, Martin AJ, Sievers R, Gerstenfeld EP. Utility of high-resolution electroanatomic mapping of the left ventricle using a multispline basket catheter in a swine model of chronic myocardial infarction. Heart Rhythm2015; 12: 144-154

4. Anter E, Tschabrunn CM, Buxton AE, Josephson ME. High-resolution mapping of postinfarction reentrant ventricular tachycardia: electrophysiological characterization of the circuit. Circulation. 2016; 134: 314-27.

5. Latcu DG, Bun SS, Saoudi N. Combined remote magnetic navigation and ultra-high-density mapping (Rhythmia ${ }^{\mathrm{TM}}$ ) in slow pathway ablation. Europace 2016; 18: 814. doi: 10.1093/europace/euv459.

6. Hooks DA, Yamashita S, Capellino S, Cochet H, Jais $\mathrm{P}$, Sacher F. Ultra-rapid epicardial activation mapping during ventricular tachycardia using continuous sampling from a high-density basket (Orion(TM)) catheter. J Cardiovasc Electrophysiol 2015; 26: 1153-4.

7. Anter E, McElderry TH, Contreras-Valdes FM, Li J, Tung P, Leshem E, Haffajee CI, Nakagawa H, Josephson ME. Evaluation of a novel high resolution mapping technology for ablation of recurrent scar-related atrial tachycardias. Heart Rhythm 2016; 13: 2048-55.

8. Steinberg BA,Piccini JP. High-density mapping of the tachycardia circuit in atrioventricular nodal reentrant tachycardia. Heart Rhythm Case Reports 2016; 2 :451453 ; DOI: http://dx.doi.org/10.1016/j.hrcr.2016.02.006 